\title{
DIVERSIDAD DEL MICROFITOPLANCTON EN LAS AGUAS OCEÁNICAS ALREDEDOR DE CUBA
}

\author{
Sandra Loza Álvarez ${ }^{1}$ y Gladys Margarita Lugioyo Gallardo ${ }^{1 *}$
}

\begin{abstract}
RESUMEN
Se evalúa la diversidad de la comunidad microfitoplanctónica en las aguas oceánicas alrededor de Cuba durante cuatro cruceros (febrero-marzo de 1999, julio-agosto del 2003, marzo del 2005 y agosto del 2005). Las muestras se recolectaron con botellas Nansen de $10 \mathrm{~L}$ de capacidad, a nivel subsuperficial y se concentraron mediante filtración invertida, a través de una malla de $20 \mu \mathrm{m}$ de diámetro de poro. El volumen de agua filtrado por estaciones osciló entre 5 y 10 L. Se reportan un total de 181 especies de microalgas ubicadas en las diferentes categorías taxonómicas. El microfitoplancton estuvo dominado en cuanto al número de especies por diatomeas 85 y dinoflagelados 47 , seguidas por cianobacterias con 23 especies y las dictiocofitas y primnesiofitas con 23 especies (mayormente cocolitofóridos). De las diatomeas, las familias Bacillariaceae, Chaetoceraceae y Rhizosoleniaceae aportan el mayor número de especies con los géneros Nitzschia, Chaetoceros y Rhizosolenia. En los dinoflagelados se distinguen las familias Ceratiaceae, Protoperidiniaceae y Oxytosaceae y los géneros Ceratium, Protoperidinium y Oxytoxum. Las aguas oceánicas al norte de Cuba presentan mayor diversidad de especies (136) con respecto a las del sur (103), como lo demuestra el índice de riqueza $\left(\mathrm{R}_{1}\right)$ que en el norte fue de 48.35 , mientras en el sur fue de 28.19.
\end{abstract}

Palabras claves: Microfitoplancton, diversidad, taxonomía, aguas oceánicas, Cuba.

\begin{abstract}
The structure of the microphytoplankton community was evaluated in oceanic waters around Cuba during four cruises (February-March 1999, July-August 2003, March 2005 and August 2005). Samples were collected with $10 \mathrm{~L}$ Nasen bottles sub-surface level and were concentrated by inverse filtration through a $20 \mu \mathrm{m}$ mesh-size net. The volume of filtered water per station ranged from 5-10 L. A total of 181 species of microalgae belonging to different taxonomic categories are reported. The microphytoplankton community, in terms of number of species, was dominated by diatoms (85) and dinoflagellates (47). Cianobacteria contributed 23 species and the dictiocophytes and primnesiophytes 23 species (mostly coccolithophorids). Among diatoms, the families Bacillariaceae, Chaetoceraceae and Rhizosoleniaceae contributed the greater number of species in the genera Nitzschia, Chaetoceros and Rhizosolenia. In dinoflagellates, the families Ceratiaceae, Protoperidiniaceae, and Oxytosaceae, and the genera Ceratium, Protoperidinium, and Oxytoxum are emphasized. The oceanic waters north of Cuba presented a greater diversity of species (136) compared to those in the south (103) as demonstrated by a higher richness index (48.35) in the north versus the one found in the south (28.19).
\end{abstract}

Keywords: Microphytoplankton, diversity, taxonomy, oceanic waters, Cuba.

1 Instituto de Oceanología, Ave. 1ra., No. 18406, entre 184 y 186, Reparto Flores, Ciudad Habana, Cuba.E-mails: sandra@oceano.inf.cu,margarita@oceano.inf.cu*

Recibido 8-I-2009

Aceptado 16-IX-2009

DOI: http://dx.doi.org/10.15359/revmar.1.2

Rev. Mar. y Cost. ISSN 1659-455X. Vol. 1. 29-47, Diciembre 2009. 


\section{INTRODUCCIÓN}

En el Golfo de México y el Mar Caribe, entre 1960 y 1980, se realizaron diversas investigaciones oceanológicas integrales para el conocimiento de las características hidrológicas, hidroquímicas, planctonológicas y pesqueras de la región (Okolodkov, 2003). En estos estudios se caracterizó el fitoplancton marino a partir de la composición taxonómica, fundamentalmente, en muestras de red (Ivanov, 1966; Khromov, 1967; Zernova, 1970; López-Baluja y Vinogradova, 1972; Zernova, 1974; Zernova y Krylov, 1974; Zernova, 1975; López-Baluja, 1976).

En estas investigaciones se inventariaron alrededor de 700 especies y variedades con su distribución geográfica y se realizó el análisis taxonómico comparativo de los géneros y especies de las regiones del Golfo de México, el Mar Caribe y el Estrecho de la Florida, encontrándose en el Golfo de México la mayor diversidad (Roujiyaynen et al. 1971; Margalef, 1978). Del total de especies reportadas en las tres regiones, 299 fueron diatomeas, 235 dinoflagelados, 90 crisofitas y primnesiofitas (mayormente cocolitofóridos), 52 cianofitas y 13 especies y variedades de clorofíceas, euglenofíceas y prasinofíceas (Okolodkov, 2003).

La región del Golfo de México se encuentra más estudiada hasta la actualidad, en cuanto a la diversidad del fitoplancton marino y su relación con la dinámica de las aguas oceánicas (Hernández-Becerril, 1995; Licea et al. 1995; Moreno et al. 1996; Aké-Castillo et al. 1999; Hernández-Becerril y Bravo-Sierra, 2000; Hernández-Becerril, 2003).

En las aguas oceánicas adyacentes a Cuba, entre las décadas de los 60 y $\operatorname{los} 80$ se realizaron algunas investigaciones sobre la diversidad del fitoplancton por López-Baluja y Vinogradova, 1972; López-Baluja, 1976; López-Baluja et al. 1985 y Popowski y Campos, 1987. Sin embargo, en las últimas décadas, se carece de una información más completa sobre la composición taxonómica del microfitoplancton.

El objetivo del presente trabajo fue describir la composición taxonómica de la fracción microfitoplanctónica $(>20 \mu \mathrm{m})$ existente en las aguas oceánicas alrededor de Cuba durante cruceros realizados en diferentes épocas de los años 1999, 2003 y 2005.

\section{MATERIALES Y MÉTODOS}

Las muestras fueron recolectadas en las aguas oceánicas al sur y al norte de Cuba, durante cruceros de investigación realizados en invierno (febrero-marzo de 1999 y marzo del 2005) y en verano (julio-agosto del 2003 y agosto del 2005) (Figura 1). 
Figura 1

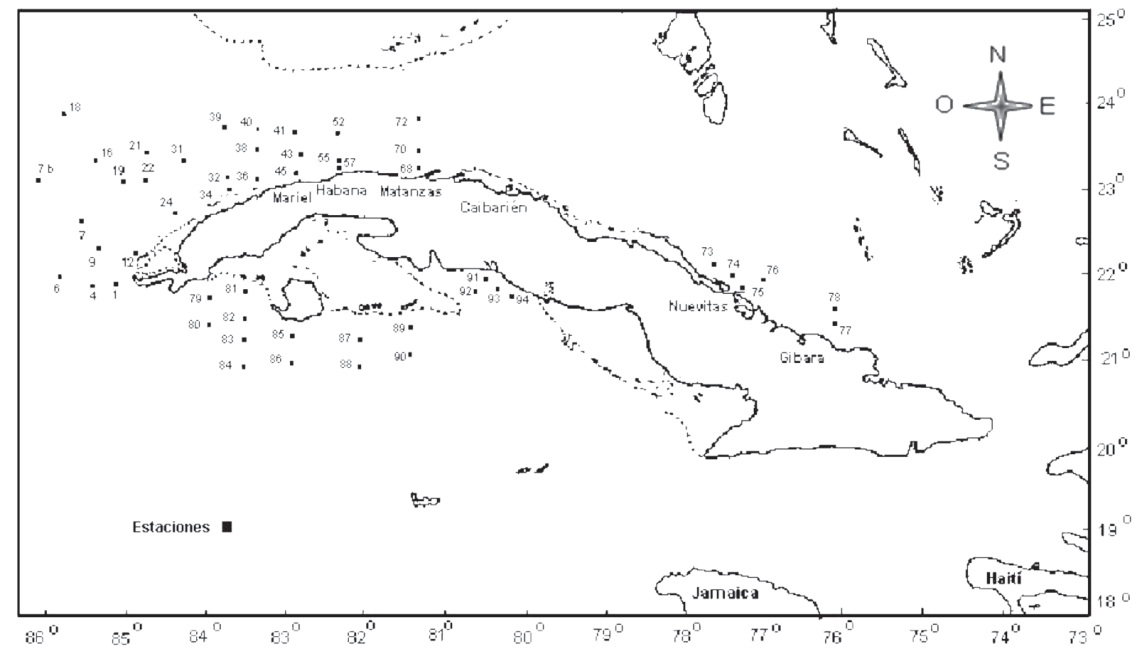

Ubicación de las estaciones de muestreo en las aguas oceánicas adyacentes a Cuba.

Location of sampling stations in oceanic waters around Cuba.

Para el análisis cualitativo del fitoplancton mayor de $20 \mu \mathrm{m}$ las muestras se recolectaron con botellas Nansen de $10 \mathrm{~L}$ de capacidad a nivel subsuperficial y se concentraron mediante filtración invertida (Sorokin, 1979) a través de una malla de $20 \mu \mathrm{m}$ de diámetro de poro. El volumen de agua filtrado por estaciones osciló entre 5 y $10 \mathrm{~L}$. Las muestras concentradas por filtración invertida se fijaron con lugol ácido y se centrifugaron.

El análisis al microscopio se realizó a partir de tres réplicas de $0.02 \mathrm{ml}$ cada una, de las que se escogieron 28 líneas al azar empleando una cámara Sedgewick-Rafter.

Para establecer las diferentes categorías taxonómicas se adoptó el sistema de clasificación de Chrétiennot-Dinet et al. (1993) y Throndsen (1993) y para su actualización se consultó el sitio algaebase. org (Guiry y Guiry, 2009). La identificación de las especies se realizó según Tomas (1993), Licea et al. (1995), Moreno et al. (1996) y Sant'Anna et al. (2005).

Se calculó el índice de riqueza de especies $\left(\mathrm{R}_{1}\right)$ para las aguas oceánicas al norte y al sur de Cuba empleando el índice de Margalef (1951) o índice de Gleason (Frontier y Pichod-Viale, 1991), modificado mediante la sustitución del área muestreada por el número de estaciones muestreadas.

\section{RESULTADOS}

Se identificaron un total de 181 especies (Cuadro 1), de las cuales 57 fueron registradas en las aguas oceánicas de las regiones norte y sur, 79 se encontraron únicamente al norte y 44 sólo en el sur (Cuadro 1). 


\section{Cuadro 1}

Lista de especies del microfitoplancton en las aguas oceánicas adyacentes a Cuba.

\section{Table 1}

List of microphytoplankton species in oceanic waters around Cuba.

\begin{tabular}{|c|c|c|}
\hline & Sur & Norte \\
\hline \multicolumn{3}{|l|}{ Phylum CYANOBACTERIA Stainer ex Cavalier-Smith } \\
\hline \multicolumn{3}{|l|}{ Clase Cyanophyceae Schaffner } \\
\hline \multicolumn{3}{|l|}{ Orden Chroococcales von Wettstein, von Westerheim } \\
\hline \multicolumn{3}{|l|}{ Familia Chroococcaceae Raberhorst } \\
\hline Chroococcus minor (Kützing) Nägeli & & $\mathbf{X}$ \\
\hline Chroococcus turgidus (Kützing) Nägeli & $\mathbf{X}$ & $\mathbf{X}$ \\
\hline Chroococcus sp Nägeli & $\mathbf{X}$ & $\mathbf{X}$ \\
\hline Coccochloris sp Sprengel & $\mathbf{X}$ & $\mathbf{X}$ \\
\hline \multicolumn{3}{|l|}{ Familia Cyanobacteriaceae } \\
\hline Aphanothece elabens (Brébisson) Elenkin & $\mathbf{X}$ & $\mathbf{X}$ \\
\hline Aphanothece sp Nägeli & & $\mathbf{X}$ \\
\hline \multicolumn{3}{|l|}{ Familia Merismopediceae Elenkin } \\
\hline Merismopedia sp Meyen & & $\mathbf{X}$ \\
\hline \multicolumn{3}{|l|}{ Familia Microcystaceae Elenkin } \\
\hline Microcystis aeruginosa (Kützing) Kützing & & $\mathbf{X}$ \\
\hline Anacystis sp Meneghini & & $\mathbf{X}$ \\
\hline Gloeocapsa sp Kützing & $\mathbf{X}$ & $\mathbf{X}$ \\
\hline \multicolumn{3}{|l|}{ Orden Nostocales Cavelier-Smith } \\
\hline \multicolumn{3}{|l|}{ Familia Nostocaceae Eichler } \\
\hline Richelia intracellularis Schmidt & $\mathbf{X}$ & $\mathbf{X}$ \\
\hline Anabaena sp Bory de Saint-Vincent ex Bornet \& Flahault & $\mathbf{X}$ & \\
\hline \multicolumn{3}{|l|}{ Orden Oscillatoriales Cavelier-Smith } \\
\hline \multicolumn{3}{|l|}{ Familia Oscillatoriaceae Engler } \\
\hline Oscillatoria margaritifera Kützing ex Gomont & $\mathbf{X}$ & \\
\hline Oscillatoria sancta f. tenuis (Woronichin) Elenkin & $\mathbf{X}$ & $\mathbf{X}$ \\
\hline Oscillatoria sp Vaucher ex Gomont & & $\mathbf{X}$ \\
\hline \multicolumn{3}{|l|}{ Familia Phormidiaceae } \\
\hline Phormidium mucicola Huber-Pestalozzi \& Naumann & $\mathbf{X}$ & $\mathbf{X}$ \\
\hline Spirulina major Kützing & $\mathbf{X}$ & $\mathbf{X}$ \\
\hline Spirulina nodosa Schiller & $\mathbf{X}$ & \\
\hline Spirulina princeps West \& West & $\mathbf{X}$ & $\mathbf{X}$ \\
\hline Spirulina subsalsa Örsted & $\mathbf{X}$ & \\
\hline Trichodesmium thiebautii Gomont ex Gomont & $\mathbf{X}$ & $\mathbf{X}$ \\
\hline \multicolumn{3}{|l|}{ Orden Synechococcales } \\
\hline \multicolumn{3}{|l|}{ Familia Synechococcaceae Komárek \& Anagnostidis } \\
\hline Synechococcus elongatus (Nägeli) Nägeli & $\mathbf{X}$ & \\
\hline Synechococcus sp Nägeli & $\mathbf{X}$ & $\mathbf{X}$ \\
\hline \multicolumn{3}{|l|}{ Phylum BACILLARIOPHYTA Engler \& Gilg } \\
\hline & Sur & Norte \\
\hline
\end{tabular}




\begin{tabular}{|c|c|c|}
\hline Clase Bacillariophyceae Haeckel & & \\
\hline Orden Bacillariales Hendey & & \\
\hline Familia Bacillariaceae Ehrenberg & & \\
\hline Bacillaria paradoxa Gmelin & & $\mathbf{X}$ \\
\hline Nitzschia elegantula Grunow & & $\mathbf{X}$ \\
\hline Nitzschia longissima (Brébisson in Kützing) Ralfs & $\mathbf{X}$ & $\mathbf{X}$ \\
\hline Nitzschia marina Grunow & $\mathbf{X}$ & \\
\hline Nitzschia oceanica Hasle & & $\mathbf{X}$ \\
\hline Nitzschia sigma (Kützing) Smith & & $\mathbf{X}$ \\
\hline Nitzschia sp Hassall & $\mathbf{X}$ & $\mathbf{X}$ \\
\hline Orden Lyrellales Mann & & \\
\hline Familia Lyrellaceae Mann & & \\
\hline Lyrella lyra (Ehrenberg) Karajeva 1978 & $\mathbf{X}$ & \\
\hline Orden Naviculales Bessey & & \\
\hline Familia Naviculaceae Kützing & & \\
\hline Navicula ovalis Hilse & & $\mathbf{X}$ \\
\hline Navicula lanceolata (Agardh) Ehrenberg & $\mathbf{X}$ & \\
\hline Navicula rostrata Ehrenberg & & $\mathbf{X}$ \\
\hline Navicula sp Bory de Saint-Vincent & $\mathbf{X}$ & $\mathbf{X}$ \\
\hline Haslea wawrikae (Hustedt) Simonsen & & $\mathbf{X}$ \\
\hline Familia Pinnulariaceae Mann & & \\
\hline Pinnularia bigibba Gaisser \& Johansen & $\mathbf{X}$ & \\
\hline Pinnularia sp Ehrenberg & & $\mathbf{X}$ \\
\hline Familia Pleurosigmataceae Mereschowsky & & \\
\hline Gyrosigma sp Hassall & $\mathbf{X}$ & $\mathbf{X}$ \\
\hline Orden Surirellales Mann & & \\
\hline Familia Surirellaceae Kützing & & \\
\hline Surirella sp Turpin & & $\mathbf{X}$ \\
\hline Clase Coscinodiscophyceae Round \& Crawford & & \\
\hline Orden Asterolamprales Round & & \\
\hline Familia Asterolampraceae Smith, Smith & & \\
\hline Asterolampra marylandica Ehrenberg & & $\mathbf{X}$ \\
\hline Asteromphalus sp. Ehrenberg & & $\mathbf{X}$ \\
\hline Orden Biddulphiales & & \\
\hline Familia Biddulphiaceae Kützing & & \\
\hline Biddulphia biddulphiana (Smith) Boyer & $\mathbf{X}$ & \\
\hline Orden Chaetocerotales Round \& Crawford & & \\
\hline Familia Chaetoceraceae Ralfs & & \\
\hline Bacteriastrum delicatulum Cleve & & $\mathbf{X}$ \\
\hline Bacteriastrum hyalinum Lauder & $\mathbf{X}$ & $\mathbf{X}$ \\
\hline Bacteriastrum $\mathrm{sp}$ Shadbolt & & $\mathbf{X}$ \\
\hline Chaetoceros affine Lauder & $\mathbf{X}$ & $\mathbf{X}$ \\
\hline Chaetoceros atlanticum Cleve & & $\mathbf{X}$ \\
\hline Chaetoceros curvisetus Cleve & & $\mathbf{X}$ \\
\hline Chaetoceros decipiens Cleve & $\mathbf{X}$ & $\mathbf{X}$ \\
\hline & Sur & Norte \\
\hline
\end{tabular}




\begin{tabular}{|c|c|c|}
\hline Chaetoceros decipiens $f$. singularis Gran & & $\mathbf{X}$ \\
\hline Chaetoceros delicatulum Ostenfeld & $\mathbf{X}$ & \\
\hline Chaetoceros didymum Ehrenberg & & $\mathbf{X}$ \\
\hline Chaetoceros diversum Cleve & $\mathbf{X}$ & $\mathbf{X}$ \\
\hline Chaetoceros lauderi Ralfs & & $\mathbf{X}$ \\
\hline Chaetoceros lorenzianum Grunow & $\mathbf{X}$ & $\mathbf{X}$ \\
\hline Chaetoceros messanense Castracane & & $\mathbf{X}$ \\
\hline Chaetoceros muelleri Lemmermann & & $\mathbf{X}$ \\
\hline Chaetoceros pelagicus f. pelagicus Gran & $\mathbf{X}$ & $\mathbf{X}$ \\
\hline Chaetoceros peruvianus Brightwell & & $\mathbf{X}$ \\
\hline Chaetoceros sp Ehrenberg & $\mathbf{X}$ & $\mathbf{X}$ \\
\hline \multicolumn{3}{|l|}{ Orden Corethrales Round \& Crawford } \\
\hline \multicolumn{3}{|l|}{ Familia Corethraceae Lebour } \\
\hline Corethron cryophilum Castracane & & $\mathbf{X}$ \\
\hline \multicolumn{3}{|l|}{ Orden Coscinodiscales Round } \\
\hline \multicolumn{3}{|l|}{ Familia Coscinodiscaceae Kützing 1844} \\
\hline Coscinodiscus sp. Ehrenberg & $\mathbf{X}$ & \\
\hline \multicolumn{3}{|l|}{ Orden Hemiaulales Round \& Crawford } \\
\hline \multicolumn{3}{|l|}{ Familia Hemiaulaceae Heiberg } \\
\hline Cerataulina pelagica (Cleve) Hendey & $\mathbf{X}$ & $\mathbf{X}$ \\
\hline Climacodium frauenfeldianum Grunow & & $\mathbf{X}$ \\
\hline Hemiaulus hauckii Grunow ex Van Heurck & $\mathbf{X}$ & $\mathbf{X}$ \\
\hline Hemiaulus membranaceus Cleve & & $\mathbf{X}$ \\
\hline Hemiaulus sinensis Greville & & $\mathbf{X}$ \\
\hline Cerataulina sp Peragallo ex Schütt & & $\mathbf{X}$ \\
\hline Eucampia sp. Ehrenberg & & $\mathbf{X}$ \\
\hline \multicolumn{3}{|l|}{ Orden Leptocylindrales Round \& Crawford } \\
\hline \multicolumn{3}{|l|}{ Familia Leptocylindraceae Lebour } \\
\hline Leptocylindrus danicus Cleve & & $\mathbf{X}$ \\
\hline Leptocylindrus mediterraneus (Perigallo) Hasle & & $\mathbf{X}$ \\
\hline Leptocylindrus sp. Cleve & & $\mathbf{X}$ \\
\hline \multicolumn{3}{|l|}{ Orden Meloseirales Crawford } \\
\hline \multicolumn{3}{|l|}{ Familia Melosiraceae Kützing } \\
\hline Hyalodiscus elegans Strelnikova & & $\mathbf{X}$ \\
\hline Melosira normanii Arnott ex Van Heurck & $\mathbf{X}$ & \\
\hline Melosira sp Agardh & & $\mathbf{X}$ \\
\hline \multicolumn{3}{|l|}{ Orden Rhizosoleniales } \\
\hline \multicolumn{3}{|l|}{ Familia Rhizosoleniaceae De Toni } \\
\hline Dactyliosolen fragilissimus (Bergon) Hasle & & $\mathbf{X}$ \\
\hline Guinardia delicatula (Cleve) Hasle & $\mathbf{X}$ & $\mathbf{X}$ \\
\hline Guinardia flaccida (Castracane) Peragallo & $\mathbf{X}$ & \\
\hline Proboscia alata (Brightwell) Sündström & & $\mathbf{X}$ \\
\hline Pseudosolenia calcar-avis (Schultze) Sündström & & $\mathbf{X}$ \\
\hline Rhizosolenia bergonii Peragallo & & $\mathbf{X}$ \\
\hline \multirow[t]{2}{*}{ Rhizosolenia clevei var communis Sündström } & & $\mathbf{X}$ \\
\hline & Sur & Norte \\
\hline
\end{tabular}




\begin{tabular}{|c|c|c|}
\hline Rhizosolenia hebetata Bailey & & $\mathbf{X}$ \\
\hline Rhizosolenia pungens (Cleve-Euler) Brunel & & $\mathbf{X}$ \\
\hline Rhizosolenia sigma Shütt & & $\mathbf{X}$ \\
\hline Dactiliosolen sp Castracane & $\mathbf{X}$ & $\mathbf{X}$ \\
\hline Rhizosolenia sp Brightwell & & $\mathbf{X}$ \\
\hline \multicolumn{3}{|l|}{ Orden Thalassiosirales } \\
\hline \multicolumn{3}{|l|}{\begin{tabular}{|l|l|} 
Familia Thallassiosiraceae \\
\end{tabular}} \\
\hline Thalassiosira subtilis (Ostenfeld) Gran & & $\mathbf{X}$ \\
\hline Thalassiosira sp Cleve & & $\mathbf{X}$ \\
\hline \multicolumn{3}{|l|}{ Familia Skeletonemaceae } \\
\hline Skeletonema costatum (Greville) Cleve & & $\mathbf{X}$ \\
\hline Streptotheka sp Greville & $\mathbf{X}$ & $\mathbf{X}$ \\
\hline \multicolumn{3}{|l|}{ Clase Fragilariophyceae Round } \\
\hline \multicolumn{3}{|l|}{ Orden Fragilariales Silva } \\
\hline \multicolumn{3}{|l|}{ Familia Fragilariaceae Greville } \\
\hline Asterionellopsis glaciales (Castracane) Round & $\mathbf{X}$ & $\mathbf{X}$ \\
\hline Asterionella notata Grunow ex Van Heurck & & $\mathbf{X}$ \\
\hline Diatoma vitrea Kützing & & $\mathbf{X}$ \\
\hline Fragilaria sp Lyngbye & & $\mathbf{X}$ \\
\hline Synedra sp Ehrenberg & $\mathbf{X}$ & $\mathbf{X}$ \\
\hline \multicolumn{3}{|l|}{ Orden Licmophorales Round, Round } \\
\hline \multicolumn{3}{|l|}{ Familia Licmophoraceae Kützing } \\
\hline Licmophora argenescens Agarth & & $\mathbf{X}$ \\
\hline Licmophora gracilis (Ehrenberg) Grunow & $\mathbf{X}$ & \\
\hline Licmophora sp Agardh & & $\mathbf{X}$ \\
\hline \multicolumn{3}{|l|}{ Orden Rhabdonematales Round \& Crawford } \\
\hline \multicolumn{3}{|l|}{ Familia Rhabdonemataceae Round \& Crawford } \\
\hline Rhabdonema adriaticum Kützing & & $\mathbf{X}$ \\
\hline \multicolumn{3}{|l|}{ Orden Striatellales Round, Round } \\
\hline \multicolumn{3}{|l|}{ Familia Striatellaceae Kützing } \\
\hline Grammatophora marina (Lyngbye) Kützing & & $\mathbf{X}$ \\
\hline \multicolumn{3}{|l|}{ Orden Thalassionemales Round } \\
\hline \multicolumn{3}{|l|}{ Familia Thalassionemataceae Round } \\
\hline Thalassionema nitzschiodes (Grunow) Mereschkowsky & & $\mathbf{X}$ \\
\hline Thalassiothrix longissima Cleve \& Grunow & & $\mathbf{X}$ \\
\hline Thalassionema sp Peragallo & $\mathbf{X}$ & $\mathbf{X}$ \\
\hline Thalassiothrix sp Cleve \& Grunow & & $\mathbf{X}$ \\
\hline \multicolumn{3}{|l|}{ Orden Toxariales Round } \\
\hline \multicolumn{3}{|l|}{ Familia Toxariaceae Round } \\
\hline Toxarium undulatum Bailey & $\mathbf{X}$ & \\
\hline \multicolumn{3}{|l|}{ Phylum CHLOROPHYTA Pascher } \\
\hline \multicolumn{3}{|l|}{ Clase Chlorophyceae Wille } \\
\hline \multicolumn{3}{|l|}{ Orden Volvocales Oltmanns } \\
\hline \multicolumn{3}{|l|}{ Familia Chlamydomonadaceae Stein } \\
\hline \multirow[t]{2}{*}{ Chlamydomona coccoides Butcher } & $\mathbf{X}$ & \\
\hline & Sur & Norte \\
\hline
\end{tabular}




\begin{tabular}{|c|c|c|}
\hline Phylum HAPTOPHYTA Hibberd ex Eduardsen \& Eikren & & \\
\hline Clase Haptophyta incertae sedis & & \\
\hline Orden Haptophyta incertae sedis & & \\
\hline Familia Calyptrosphaeraceae Boudreaux \& Hay & & \\
\hline Calyptosphaera oblonga Lohmann & $\mathbf{X}$ & \\
\hline Syracolithus dalmaticus (Kamptner) Loeblich Jr. \& Tappan & $\mathbf{X}$ & $\mathbf{X}$ \\
\hline Clase Prymnesiophyceae Hibberd & & \\
\hline Orden Coccolithales Schwarz & & \\
\hline Familia Calcidiseaceae Young \& Brown & & \\
\hline Oolithotus fragilis (Lohmann) Martini \& Müller & $\mathbf{X}$ & \\
\hline Umbilicosphaera sibogae (Weber-van Bosse) Gaarder & $\mathbf{X}$ & $\mathbf{X}$ \\
\hline Familia Coccolithaceae Poche & & \\
\hline Coccolithus pelagicus (Wallich) Schiller & $\mathbf{X}$ & $\mathbf{X}$ \\
\hline Orden Isochrysidales Pascher & & \\
\hline Familia Noëlaerhabdacae Jerkovic & & \\
\hline Emiliania huxleyi (Lochmann) Hay \& Mohler & $\mathbf{X}$ & $\mathbf{X}$ \\
\hline Orden Phaeocystales Medlin & & \\
\hline Familia Phaeocystaceae Lagerheim & & \\
\hline Phaeocystis sp Lagerheim & & $\mathbf{X}$ \\
\hline Orden Prymnesiales Papenfuss & & \\
\hline Familia Prymnesiaceae Conrad ex Schmidt & & \\
\hline Chrysochromulina sp Lackey & $\mathbf{X}$ & \\
\hline Orden Syracosphaerales Hay & & \\
\hline Familia Calciosoleniaceae Kamptner & & \\
\hline Calciosolenia sp Gran & $\mathbf{X}$ & $\mathbf{X}$ \\
\hline Familia Rhabdosphaeraceae Lemmermann & & \\
\hline Discophaera tubifera (Murray \& Blackman) Ostenfeld & $\mathbf{X}$ & $\mathbf{X}$ \\
\hline Rhabdosphaera stylifer Lohmann & $\mathbf{X}$ & \\
\hline Discophaera sp Haeckel & $\mathbf{X}$ & $\mathbf{X}$ \\
\hline Familia Syracosphaeraceae (Lohmann) Lemmermann & & \\
\hline Michaelsarsia elegans Gran & $\mathbf{X}$ & \\
\hline Syracosphaera corii Schiller & $\mathbf{X}$ & $\mathbf{X}$ \\
\hline Syracosphaera pulchra Lohmann & $\mathbf{X}$ & \\
\hline Michaelsarsia sp Gran & $\mathbf{X}$ & \\
\hline Syracosphaera sp Lohmann & $\mathbf{X}$ & $\mathbf{X}$ \\
\hline Orden Zygodiscales Joung \& Bown & & \\
\hline Familia Pontosphaeraceae Lemmermann & & \\
\hline Pontosphaera borealis Ostenfeld & $\mathbf{X}$ & $\mathbf{X}$ \\
\hline Scythosphaera apsteini Lohmann & $\mathbf{X}$ & \\
\hline Pontosphaera sp Lohmann & $\mathbf{X}$ & \\
\hline Phylum HETEROKONTOPHYTA Moestrup & & \\
\hline Clase Cryptophyceae Fritsch & & \\
\hline Orden Cryptomonadales Pascher & & \\
\hline Familia Chryptomonadaceae Ehrenberg & & \\
\hline Cryptomona sp Ehrenberg & & $\mathbf{X}$ \\
\hline & Sur & Norte \\
\hline
\end{tabular}




\begin{tabular}{|c|c|c|}
\hline Clase Dictyochophyceae Silva & & \\
\hline Orden Dictyochales Haeckel & & \\
\hline Familia Dictyochaceae Lemmermann & & \\
\hline Dictyocha fibula Ehrenberg & $\mathbf{X}$ & $\mathbf{X}$ \\
\hline Distephanus specullum (Ehrenberg) Haeckel & & $\mathbf{X}$ \\
\hline Mesocena polimorpha Lemmermann & $\mathbf{X}$ & \\
\hline Clase Raphidophyceae Chadefaud ex Silva & & \\
\hline Orden Chattonellales Throndsen & & \\
\hline Familia Chattonellaceae Throndsen & & \\
\hline Chattonella antiqua (Hada) Ono & & $\mathbf{X}$ \\
\hline Phylum MYZOZOA Cavalier-Smith \& Chao & & \\
\hline Clase Dinophyceae Fritsch & & \\
\hline Orden Gymnodiniales Apstein & & \\
\hline Familia Gymnodiniaceae Lankester & & \\
\hline Amphidinium crassum Lohmann & & $\mathbf{X}$ \\
\hline Gymnodinium sanguineum Hirasaka & & $\mathbf{X}$ \\
\hline Amphidinium sp Claparède \& Lachmann & $\mathbf{X}$ & $\mathbf{X}$ \\
\hline Gymnodinium sp Stein & $\mathbf{X}$ & $\mathbf{X}$ \\
\hline Gyrodinium sp Kofoid \& Swery & $\mathbf{X}$ & $\mathbf{X}$ \\
\hline Orden Peridiniales Haeckel & & \\
\hline Familia Ceratiaceae Kofoid & & \\
\hline Ceratium arietinum Cleve & & $\mathbf{X}$ \\
\hline Ceratium breve (Ostenfeld \& Smidt) Schröder & $\mathbf{X}$ & \\
\hline Ceratium buceros (Zacharias) Schiller & & $\mathbf{X}$ \\
\hline Ceratium furca var. furca (Ehrenberg) Jorgensen & $\mathbf{X}$ & $\mathbf{X}$ \\
\hline Ceratium fusus (Ehrenberg) Dujardin & $\mathbf{X}$ & \\
\hline Ceratium longissimum (Schröder) Kofoid & $\mathbf{X}$ & \\
\hline Ceratium pentagonum Gourret & $\mathbf{X}$ & \\
\hline Ceratium pulchellum Schröder & $\mathbf{X}$ & $\mathbf{X}$ \\
\hline Ceratium teres Kofoid & $\mathbf{X}$ & $\mathbf{X}$ \\
\hline Ceratium sp Schrank & & $\mathbf{X}$ \\
\hline Familia Ceratocoryaceae Lindemann & & \\
\hline Ceratocorys horrida Stein & $\mathbf{X}$ & $\mathbf{X}$ \\
\hline Familia Gonyaulacaceae Lindemann & & \\
\hline Gonyaulax kofoidii Pavillard & $\mathbf{X}$ & \\
\hline Gonyaulax polygramma Stein & $\mathbf{X}$ & \\
\hline Gonyaulax spinifera (Claparède \& Lachmann) Diesing & $\mathbf{X}$ & \\
\hline Gonyaulax sp. Diesing & $\mathbf{X}$ & \\
\hline Familia Oxytoxaceae Lindemann & & \\
\hline Corythodinium diploconus (Stein) Taylor & $\mathbf{X}$ & \\
\hline Oxytoxum laticeps Schiller & & $\mathbf{X}$ \\
\hline Oxytoxum reticulatum (Stein) Schütt & & $\mathbf{X}$ \\
\hline Oxytoxum scolopax Stein & & $\mathbf{X}$ \\
\hline Oxytoxum turbo Kofoid & & $\mathbf{X}$ \\
\hline Oxytoxum variabile Schiller & $\mathbf{X}$ & \\
\hline & Sur & Norte \\
\hline
\end{tabular}




\begin{tabular}{|c|c|c|}
\hline Corythodinium sp. Loeblich \& Loeblich III & $\mathbf{X}$ & $\mathbf{X}$ \\
\hline Oxytoxum sp Stein & & $\mathbf{X}$ \\
\hline \multicolumn{3}{|l|}{ Familia Peridiniaceae Ehrenberg } \\
\hline Glenodinium paululum Lindemann & $\mathbf{X}$ & $\mathbf{X}$ \\
\hline Peridinium aciculiferum Lemmermann & $\mathbf{X}$ & \\
\hline Peridinium quinquecorne Abé & $\mathbf{X}$ & $\mathbf{X}$ \\
\hline Glenodinium sp. Ehrenberg ex Ralfs & $\mathbf{X}$ & $\mathbf{X}$ \\
\hline \multicolumn{3}{|l|}{ Familia Podolampadaceae Lindemann } \\
\hline Podolampas palmipes Stein & $\mathbf{X}$ & \\
\hline Podolampas spinifera Okamura & $\mathbf{X}$ & $\mathbf{X}$ \\
\hline \multicolumn{3}{|l|}{ Familia Protoperidiniaceae Bujak \& Davies } \\
\hline Protoperidinium bispinum (Schiller) Balech & $\mathbf{X}$ & \\
\hline Protoperidinium oceanicum (Vanhöffen) Balech & $\mathbf{X}$ & \\
\hline Protoperidinium pyriforme (Paulsen) Balech & $\mathbf{X}$ & \\
\hline Protoperidinium sp. Bergh & $\mathbf{X}$ & $\mathbf{X}$ \\
\hline \multicolumn{3}{|l|}{ Orden Prorocentrales Lemmermann } \\
\hline \multicolumn{3}{|l|}{ Familia Prorocentraceae Stein } \\
\hline Prorocentrum compressum (Bailey) Abe ex Dodge & & $\mathbf{X}$ \\
\hline Prorocentrum gracile Schütt & & $\mathbf{X}$ \\
\hline Prorocentrum lima (Ehrenberg) Dodge & & $\mathbf{X}$ \\
\hline Prorocentrum marinum (Cienkowski) Loeblich III & $\mathbf{X}$ & $\mathbf{X}$ \\
\hline Prorocentrum micans Ehrenberg & & $\mathbf{X}$ \\
\hline Prorocentrum scutellum Schröder & $\mathbf{X}$ & \\
\hline Prorocentrum sp. Ehrenberg & $\mathbf{X}$ & $\mathbf{X}$ \\
\hline \multicolumn{3}{|l|}{ Orden Pyrocystales Apstein } \\
\hline \multicolumn{3}{|l|}{ Familia Pyrocystaceae (Schütt) Lemmermann } \\
\hline Pyrocystis fusiformis (Wyville-Thomson ex Haeckel) Blackman & $\mathbf{X}$ & \\
\hline \multicolumn{3}{|l|}{ Orden Thoracosphaerales Tangen } \\
\hline \multicolumn{3}{|l|}{ Familia Thoracosphaeraceae Schiller } \\
\hline Thoracosphaera sp Kamptner & $\mathbf{X}$ & \\
\hline
\end{tabular}

De las diatomeas, las familias Bacillariaceae, Chaetoceraceae y Rhizosoleniaceae aportan la mayor riqueza de especies distribuidas con los géneros Nitzschia, Chaetoceros y Rhizosolenia, fundamentalmente. En los dinoflagelados fueron importantes los organismos de las familias Ceratiaceae, Protoperidiniaceae y Oxytosaceae con los géneros Ceratium, Protoperidinium y Oxytoxum (Cuadro 1).

En general, se observó que del total de especies reportadas, las dia- tomeas fueron el grupo más abundante con 85 especies, seguidas por los dinoflagelados (47). Las cianobacterias contribuyeron con 23 especies y las dictiocofitas y primnesiofitas con 23 especies, con un mayor aporte de los cocolitofóridos (11\%) (Cuadro 2). 


\section{Cuadro 2}

Número total de especies identificadas por categorías taxonómicas en las aguas oceánicas adyacentes a Cuba.

\section{Table 2}

Total number of species identified, by taxonomic category, in oceanic waters around Cuba.

\begin{tabular}{cccc}
\hline $\begin{array}{c}\text { Clases } \\
\text { Taxonómicas }\end{array}$ & $\begin{array}{c}\text { Aguas } \\
\text { Oceánicas }\end{array}$ & ZEE Sur & ZEE Norte \\
\hline Fragilariophyceae & 15 & $5(4.8 \%)$ & $13(9.5 \%)$ \\
\hline Bacillariophyceae & 17 & $8(7.8 \%)$ & $13(9.5 \%)$ \\
\hline Coscinodiscophyceae & 53 & $18(17.5 \%)$ & $48(35.3 \%)$ \\
\hline Dinophyceae & 47 & $33(32 \%)$ & $29(21.3 \%)$ \\
\hline Cyanophyceae & 23 & $17(16.5 \%)$ & $18(13.2 \%)$ \\
\hline Prymnesiophyceae & 20 & $19(18.4 \%)$ & $11(8.1 \%)$ \\
\hline Dictyochophyceae & 3 & $2(1.9 \%)$ & $2(1.5 \%)$ \\
\hline Chlorophyceae & 1 & $1(0.9 \%)$ & - \\
\hline Cryptophyceae & 1 & $\mathbf{-}$ & $1(0.7 \%)$ \\
\hline Rhaphidophyceae & 1 & $\mathbf{1 0 3}$ & $\mathbf{1 3 6 . 1 9}$ \\
\hline Total & $\mathbf{1 8 1}$ & $0.7 \%)$ \\
\hline
\end{tabular}

El análisis de la riqueza de especies en el norte y el sur de la Zona Económica Exclusiva (ZEE) mostró que, la comunidad fitoplanctónica mayor de $20 \mu \mathrm{m}$ en el norte resultó más rica (136), con un valor del índice de riqueza $\left(\mathrm{R}_{1}\right)$ de 48.35 , mientras en el sur el número de especies encontradas fue menor (103), con un valor de $\mathrm{R}_{1}=28.19$ (Cuadro 2). Es de resaltar que en la ZEE norte las diatomeas tuvieron la mayor contribución (74 especies), lo que representó el $54.3 \%$ del total de las especies de la zona. Por su parte, en la ZEE sur los cocolitofóridos (Prymnesiophyceae) representaron el $18.4 \%$, mientras en el norte sólo fueron el $8.1 \%$. El resto 
de los grupos, en general, mostró un aporte similar a la diversidad en ambas regiones (Cuadro 2).

En el análisis estacional de la diversidad del microfitoplancton en la ZEE, se observó que en el norte las diatomeas fueron el grupo mejor representado, con un incremento del número de especies en el crucero de marzo de 1999 (Cuadro 3). Sin embargo, en el sur se encontró que tanto en marzo como en agosto del año 2005 fueron los dinoflagelados y las diatomeas los que más contribuyeron a la riqueza específica de la región, con un aporte similar de ambos grupos en cada una de las épocas, aunque en el invierno el número de especies de diatomeas resultó superior con respecto al verano (Cuadro 3). Por su parte, las cianobacterias mostraron una disminución en el número de especies en la época de invierno tanto en el norte como en el sur (Cuadro 3).

\section{Cuadro 3}

Número total de especies del microfitoplancton por grupos taxonómicos fundamentales en las aguas oceánicas al norte y al sur de Cuba en época de invierno y verano.

Table 3

Total number of microphytoplankton species, by fundamental taxonomic group, from oceanic waters in northern and southern Cuba during winter and summer.

\begin{tabular}{lccc}
\hline $\begin{array}{c}\text { Grupos del } \\
\text { fitoplancton }\end{array}$ & $\begin{array}{c}\text { Marzo } \\
\text { de 1999 }\end{array}$ & $\begin{array}{c}\text { Julio-Agosto } \\
\text { del 2003 }\end{array}$ & $\begin{array}{c}\text { Agosto } \\
\text { del 2005 }\end{array}$ \\
\hline Diatomeas & 31 & 23 & 20 \\
\hline Dinoflagelados & 10 & 7 & 12 \\
\hline Cianobacterias & 3 & 8 & 7 \\
\hline Otros grupos* & 5 & 7 & 3 \\
\hline
\end{tabular}




\begin{tabular}{lcc}
\hline \multicolumn{1}{c}{$\begin{array}{c}\text { Grupos del } \\
\text { fitoplancton }\end{array}$} & $\begin{array}{c}\text { Marzo } \\
\text { del 2005 }\end{array}$ & $\begin{array}{c}\text { Agosto } \\
\text { del 2005 }\end{array}$ \\
\hline Diatomeas & 18 & 13 \\
\hline Dinoflagelados & 19 & 14 \\
\hline Cianobacterias & 5 & 12 \\
\hline Otros grupos* & 16 & 6 \\
\hline
\end{tabular}

* En otros grupos se encuentran las clases Chlorophyceae, Dictyochophyceae, Prymnesiophyceae, Cryptophyceae y Raphidophyceae.

\section{DISCUSIÓN}

La diversidad del fitoplancton marino generalmente se describe y se mide en términos de riqueza de especies (John, 1994; Hernández-Becerril, 2003). La inmensa mayoría de las especies de microalgas planctónicas marinas han sido definidas como especies morfológicas, con muy poca información sobre los procesos y mecanismos de reproducción (Hernández-Becerril, 2003). Es por ello que la evaluación sobre la diversidad del microfitoplancton realizada en este estudio se basó en la separación de las especies de acuerdo con las características morfológicas de las células.

La riqueza de especies del fitoplancton mayor de $20 \mu \mathrm{m}$ en las aguas oceánicas adyacentes a Cuba y su distribución en las diferentes categorías taxonómicas resultó similar a lo informado por Popowski y Campos (1987) y Loza (2007) para la misma zona, a pesar de que se conoce únicamente un porcenta- je de las especies que existen en el medio natural (Sournia et al. 1991; Sournia, 1995; Tett y Barton, 1995) y de las discrepancias entre los autores en el número de clases taxonómicas (Chrétiennot-Dinet et al. 1993). Popowski y Campos (1987) refirieron un total de 167 especies distribuidas en 91 diatomeas, 66 dinoflagelados, 3 primnesiofíceas, 4 cianofitas, 2 crisofíceas y una clorofícea, y Loza (2007) informó un total de 151 especies, de las cuales los dinoflagelados y las diatomeas fueron los mejores representados.

Sin embargo, López-Baluja et al. (1992), a partir del análisis realizado en el período de 1965 y 1985 para el Golfo de México y el Mar Caribe, incluyendo las aguas cubanas de la plataforma insular y las oceánicas adyacentes, informan un listado de 529 especies, de ellas 264 fueron dinoflagelados, 230 diatomeas, 21 primnesiofíceas, 8 cianobacterias y 3 especies de criptofíceas y de clorofíceas. Este incremento en el número de 
especies registrado por estos autores puede estar relacionado con la mayor diversidad de especies del microfitoplancton que existe en el Golfo de México (Okolodkov, 2003), además de que también incluyen las aguas más enriquecidas de la plataforma.

Algunas de las especies de microfitoplancton encontradas en esta investigación como Ceratium furca, Rhizosolenia sp, Hemiaulus membranaceus, Chaetoceros curvisetus, Emiliania huxleyi, Oxytoxum variabile, están distribuidas principalmente en mares tropicales y algunas de ellas son características de aguas afloradas y coinciden con las reportadas por Margalef (1969) en un estudio realizado en el Mar Caribe.

El grupo de las diatomeas aportó el mayor número de especies, lo que concuerda con lo informado por Popowski y Campos (1987) para la ZEE de Cuba. Hernández-Becerril (2003) plantea que este grupo es el más conocido y diverso de las microalgas planctónicas marinas y que está distribuido en todos los mares, pero su contribución está relacionada con la disponibilidad de nutrientes.

En las aguas oceánicas de Cuba se encontraron 20 especies de cocolitofóridos, lo que concuerda con el estudio de López-Baluja et al. (1992), que encontró 21 primnesiophyta. La clase Primnesioficeae incluye un número aproximado de 280 especies, de ellas los cocolitofóridos contribuyen mayoritariamente al fitoplancton marino (Thomsen et al. 1994; Sournia, 1995). Gaarder y Hasle (1971) informan para el Golfo de México 29 taxones de cocolitofóridos; mientras Bravo-Sierra y Hernández-Becerril (2000) reportan 38 especies para el Pacífico mexicano.

Al comparar la diversidad de especies del microfitoplancton en las aguas oceánicas del norte con las del sur, se observó que en el norte la diversidad fue mayor y que en los meses correspondientes a la época de invierno, el grupo que más aportó a la riqueza de especies fue el de las diatomeas. Este resultado podría estar relacionado con el hecho de que a pesar de la oligotrofía que caracteriza a las aguas oceánicas adyacentes a Cuba, la zona norte se distingue por presentar un mayor enriquecimiento relativo de nutrientes y materia orgánica (Lugioyo, 2003), lo que al parecer favorece, en términos generales, la diversidad de especies y en particular de las diatomeas, las cuales en general presentan elevados requerimientos de nutrientes (Moreno et al. 1996).

Este enriquecimiento relativo de la ZEE norte podría estar relacionado con una mayor exportación de nutrientes desde las estrechas plataformas, si tenemos en cuenta que en esta región, el movimiento de las corrientes, por efecto del viento, provoca divergencia y afloramiento costero, que conlleva a un relativo incremento del contenido de sales minerales (Arriaza et al. 2007), además de que existen procesos de afloramientos orográficos sustentados en las elevadas con- 
centraciones de silicio (superiores a $5 \mu \mathrm{mol} . \mathrm{L}^{-1}$ ) encontradas en esta zona (Penié, 1991). Por otra parte, el tráfico continuo de embarcaciones por el Canal Viejo de las Bahamas podría favorecer también el aporte de nutrientes y materia orgánica en zonas más alejadas de la costa. Estas características de un mayor enriquecimiento relativo en las aguas al norte se refuerzan en el invierno, cuando, producto de los frentes fríos, se debilita la estratificación térmica y ocurre una entrada adicional de nutrientes proveniente de las aguas profundas.

Sin embargo, en la ZEE sur, el mayor aporte de los dinoflagelados a la riqueza de especies, tanto en el invierno como en el verano, con respecto al norte, podría estar favorecido por las características fisiológicas de este grupo, al presentar bajos requerimientos de nutrientes y una variada nutrición (autótrofa, heterótrofa y mixotrófica) (Gamboa-Márquez et al. 1994; Licea et al. 1995) que le permite adaptarse a las aguas empobrecidas en compuestos biogénicos, como son las aguas oceánicas del sur de Cuba (Fernández et al. 1990). Por otra parte, el hecho de que se haya encontrado una riqueza de especies de diatomeas similar a la de los dinoflagelados podría obedecer a la existencia en estas aguas oligotróficas de zonas puntuales enriquecidas en nutrientes, producto del intercambio con las aguas provenientes de las bahías y por el ingreso de nutrientes de las capas profundas hacia las superficiales en el invierno.

El grupo de las cianobacterias mostró una disminución del número de especies en la época de invierno tanto en el norte como en el sur; esto podría guardar relación con el hecho de que durante el invierno aumenta el oleaje y la oxigenación del agua, lo que afecta la capacidad de fijar el nitrógeno molecular que tienen las formas filamentosas de este grupo, ya que para la fijación del nitrógeno molecular se necesitan condiciones de microaerofilia (Carpenter y Price, 1976; Corredor, 1977; Sant'Anna et al. 2005).

En las cianobacterias, se distinguió la especie Trichodesmium thiebautii Gomont ex Gomont por su elevada frecuencia de aparición. López-Baluja y Vinogradova (1972) y Popowski y Campos (1987), en estudios sobre la estructura comunitaria del plancton de red en las aguas oceánicas alrededor de Cuba, también encontraron un predominio de esta especie.

\section{CONCLUSIONES}

En las aguas oceánicas adyacentes a Cuba, en el período comprendido entre 1999 y 2005, se identificaron un total de 181 especies del microfitoplancton. Las diatomeas fueron el grupo más diverso con 85 especies, seguido por los dinoflagelados con 47. Las cianobacterias contribuyeron con 23 especies y las dictiocofitas y primnesiofitas con 23 especies, con un mayor aporte de los cocolitofóridos. 
En el norte, la comunidad fitoplanctónica mayor de $20 \mu \mathrm{m}$ resultó más rica en cuanto al número de especies (136) con un valor del índice de riqueza $\left(R_{1}\right)$ de 48.35 , mientras en el sur el número de especies encontradas fue menor (103), con un valor de $R_{1}=28.19$.

En la ZEE norte, se observó durante el invierno un incremento del número de especies de diatomeas. Sin embargo, en la ZEE sur, tanto en el invierno como en el verano, fueron los dinoflagelados y las diatomeas los que más contribuyeron a la riqueza de especies de la región con un aporte similar de ambos grupos.

\section{BIBLIOGRAFÍA}

Aké-Castillo, J. A., Hernández-Becerril, D. U. \& Meave del Castillo, M. E. (1999). Species of the genus Thalassiosira (Basillariophyceae) from the Gulf of Tehuantepec. Bot. Mar., 42, 487-503.

Arriaza, L., Rodas, L., Ramírez, O., Simanca, J. \& García, R. (2007). Cartas de corrientes superficiales alrededor de Cuba a partir de la modelación numérica. (CDROOM) (ISBN 978-959-298010-5).

Bravo-Sierra, E. \& Hernández-Becerril, D. U. (2000). Coccolithophorids from various locations of the Mexican Pacific. In: Procedings 8 th International Nannoplankton Association (INA). Bremen, Alemania. 7 p.
Carpenter, E. J. \& Price, C. C. (1976). Marine Oscillatoria trichodesmium: exploration for aerobic nitrogen fixation whithout heterocysts. Sci., 191, 1278-1280.

Chrétiennot-Dinet, M. J., Sournia, A., Ricard, M. \& Billard, C. (1993). A classification of the marine phytoplankton of the World from class to genus. Phycology, 32, 159-179.

Corredor, J. E. (1977). Aspects of phytoplankton dynamics in the Caribbean sea and adjacent regions. In: Symposium on progress in marine research in the Caribbean and adjacent regions. FAO. Fish. Rep., 200, 101-114.

Fernández, M., Hidalgo, R. M., López, D., García, I. \& Penié, I. (1990). Caracterización hidroquímica de la Fosa de Jagua y la Zona Económica Exclusiva al S de Cuba. (Inédito). Informe final de tema, Archivo Científico Instituto de Oceanología. 24 p.

Frontier, S. \& Pichod-Viale, D. (1991). Ecosystémes: structure, fonctionnement, evolution. Collection d'ecologie. Masson, Paris, 21, p. 392.

Gaarder, K. R. \& Hasle, G. R. (1971). Coccolithophorids of the Gulf of Mexico. Bull. Mar. Sci., 21, 519544.

Gamboa-Márquez, J. F., Sánchez-Suárez, I. G. \& La Barbera-Sánchez, A. (1994). Dinoflagelados (Pyrrophyta) del Archipiélago Los Roques (Venezuela): Familias Prorocentraceae y Ostreopsidaceae. Acta Cient. 
Venezolana. Biol. Mar., 45, 140152.

Guiry, M. D. \& Guiry G. M. (2009). AlgaeBase. World-wide electronic publication, National University of Ireland, Galway. http:// www.algaebase.org.

Hernández-Becerril, D. U. (1995). Planktonic diatoms from the Gulf of California and coast off Baja California: the genera Rhizosolenia, Proboscia, Pseudosolenia, and former Rhizosolenia species. Diatom Res., 10, 251-267.

Hernández-Becerril, D. U. (2003). Diversidad del fitoplancton marino de México. Un acercamiento actual. Cap. 1. En: Planctonología Mexicana. Barreiro-Güemes M. T., M. E. Meave del Castillo, M. Signoret-Poillon \& M. G. Figueroa-Torres (eds.). Sociedad Mexicana de Planctonología, A. C. México, 1-18.

Hernández-Becerril, D. U. \& Bravo-Sierra, E. (2000). Cuatro nuevos registros de dinoflagelados planctónicos para el Pacífico mexicano. En: Estudios sobre Plancton en México y el Caribe. Ríos Jara, E., E. Juárez Carillo, M. Pérez Peña, E. López Uriarte, E. G. Robles Carero, D. U. Hernández-Becerril \& M. Silva Briazo (eds.). Sociedad Mexicana de Planctonología (SOMPAC) y Universidad de Guadalajara (CUCBA), 79-80.

Ivanov, A. I. (1966). Some data on the phytoplankton of the Gulf of
Mexico and the Florida strait. In: Studies on the Central American seas, 1. Kiev, 81-91.

John, D. M. (1994). Biodiversity and conservation: an algal perspective. The Phycologist, 38, 3-15.

Khromov, N. S. (1967). Studies on plankton in the Gulf of Mexico and the Caribbean Sea. In: Soviet-Cuban Fishery. Research (VNIRO-CIP), Moscow, 2, 3957.

Licea, S., Moreno, J. L., Santoyo, H. \& Figueroa, G. (1995). Dinoflagelados del Golfo de California. Universidad Autónoma de Baja California Sur. 165 p.

López-Baluja, L. (1976). Phytoplankton of Cuban waters. Synopsis of PhD Thesis in Biology, Institute of Oceanology, URSS, Academy of Sciences, Moscow.

López-Baluja, L. \& Vinogradova, L. (1972). El fitoplancton en aguas adyacentes al archipiélago cubano. Serie Oceanológica, 13, 1-24. López-Baluja, L., Zernova, V. V. \& Semina, H. J. (1985). Flora planctónica del Mediterráneo Americano. Contribuciones al Simposio de Ciencias Marinas y VII Jornada Científica del Instituto de Oceanología: XX Aniversario de la Academia de Ciencias de Cuba, Palacio de las Convenciones, La Habana, 1, 111-115.

López-Baluja, L., Zernova, V. V. \& Semina, G. I. (1992). El fitoplancton de aguas cubanas y del Golfo de México (en ruso). Nauka Press, Moscow, Rusia. 215 p. 
Loza, S. (2007). Las microalgas. En: La biodiversidad marina de Cuba. Claro, R. (ed.) (CD-Room). Instituto de Oceanología, Ministerio de Ciencia, Tecnología y Medio Ambiente, La Habana, Cuba.

Lugioyo, G. M. (2003). Distribución, relaciones tróficas y diversidad del bacterioplancton de las aguas oceánicas de Cuba. Tesis Doctoral, Universidad de La Habana, Cuba. $140 \mathrm{p}$.

Margalef, R. (1951). Diversidad de especies en las comunidades naturales. Publicación Instituto Biología Aplicada, Barcelona, 9, 5-27.

Margalef, R. (1969). El ecosistema pelágico del mar Caribe. En: $\mathrm{Me}$ moria, Fundación "La Salle", 29, 5-36.

Margalef, R. (1978). Regularidad en la distribución de la diversidad del fitoplancton en un área del mar Caribe. Invest. Pes., 36(2), 241-264.

Moreno, J. L., Licea, S. \& Santoyo, H. (1996). Diatomeas del Golfo de California Sur. Universidad Autónoma de Baja California Sur Press, México. 272 p.

Okolodkov, Y. B. (2003). Una revisión de investigaciones rusas de plancton en el Golfo de México y Mar Caribe en los años 19601980. Hidrobiol., 13(3), 207-221.

Penié, I. (1991). Caracterización hidroquímica de las aguas de la Zona Económica Exclusiva al norte de Cuba. Informe Archivo
Científico Instituto de Oceanología, Cuba. 40 p.

Popowski, C. G. \& Campos, A. (1987). Estructura comunitaria del plancton oceánico alrededor de Cuba en marzo de 1981. Rep. Invest., 64, 1-15.

Roujiyaynen, M. I., Senichkina, L. G. \& Georgieva, L. V. (1971). Review of the taxonomic composition of phytoplankton in the Central American seas. In: Studies on the Central American seas, Kiev, 3, 16-49.

Sant'Anna, C., Azevedo, M. T., Agujaro, L., Carvalho, M. C. \& Souza, R. C. (2005). Manual ilustrado para identificación y conteo de cianobacterias planctónicas de aguas continentales brasileiras. Sant'Anna C., M. T. Azevedo, L. Agujaro, M. C. Carvalho \& R. C. Souza (eds.). Interciencia Press, Sao Paulo, Brasil. 135 p.

Sorokin, Yu. I. (1979). Métodos para la concentración del fitoplancton (en ruso). Hydrobiol., 15, 71-76.

Sournia, A. (1995). Red tide and toxic marine phytoplankton of the world ocean: an inquiry into diversity. In: Harmful Marine Algal Blooms. Lassus, P., G. Arzul, E. Erand, P. Gentien \& C. marcaillou (eds.). Lavoisier, Intercept Ltd., 103-112.

Sournia, A., Chrétiennot-Dinet, M. J. \& Ricard, M. (1991). Marine phytoplankton: how many species in the world ocean? J. Plankton Res., 13, 1093-1099. 
Tett, P. \& Barton, E. D. (1995). Why are they about 5000 species of phytoplankton in the sea? $J$. Plankton Res., 17, 1693-1704.

Thomsen, H. A., Buck, K. B. \& Chavez, F. P. (1994). Haptophytes as components of marine phytoplankton. In: The haptophyte algae. Green, J. C. \& B. S. C. Leadbeater (eds). Syst. Assoc., Spec., Claredon Press, Oxford, 51, 187208.

Throndsen, J. (1993). The planktonic marine flagellates. In: Marine phytoplankton: a guide to naked flagellates and coccolothophorids. Tomas, C. R. (ed.). Academic Press, 7-145.

Tomas, C. R. (1993). Marine phytoplankton: a guide to naked flagellates and coccolothophorids. Academic Press, London.
Zernova, V. V. (1970). Acerca de las algas planctónicas del Golfo de México y el Mar Caribe (en ruso). Okeanology Issled., Moscú, 20, 69-104.

Zernova, V. V. (1974). Species structure of phytocene in the southern Gulf of Mexico. In: Soviet- Cuban Fishery Research (VNIRO-CIP), Moscow, 4, 117-131.

Zernova, V.V.(1975). Phytoplankton in some areas of the Caribbean Sea and the Gulf of Mexico. Proceeding of the Institute of Oceanology. URSS Academy of Sceinces, 101, 41-51.

Zernova, V. V. \& Krylov, V. V. (1974). Species of unicellular algae new to the Gulf of Mexico and the Caribbean Sea. In: Soviet-Cuban Fishery Research (VNIRO-CIP), 2, Moscow, 132-134. 